\title{
Digitaliser le doigt. Les écrans tactiles rêvent-ils de viande humaine?
}

\section{Digitalize the finger. Do touch screens dream about human flesh?}

Inès Garmon

GRIPIC (CELSA - Paris Sorbonne), 77 rue de Villiers, 92100 Neuilly-sur-Seine

Résumé. Cet article propose de dénaturaliser la notion de «tactile» et le vocabulaire métaphorique qui l'accompagne - telle que la « fluidité », l'« interactivité » ou la « reponsivité » - qui engagent un rapport fasciné à l'écran contemporain. Nous nous intéressons pour cela au coup de doigt sur l'écran, c'est-à-dire au moment où l'usager manipule du bout du doigt l'interface : c'est dans cette rencontre entre du technique et du corporel que se joue la continuité entre le corps et l'écran. Il s'agit d'observer les manières dont ce doigt est digitalisé, traité par l'écran; d'interroger le tactile à la fois comme processus technique, informatique ; humain, sémiotique et anthropologique ; et économique, typique d'une industrie nouvelle. Dans un premier temps, nous reviendrons sur les conditions d'élaboration de la critique de la notion de « tactile » et l'outillage conceptuel qu'elle demande, celui de l'écriture. Nous proposons de réinscrire l'écran d'abord dans sa dimension technique, ergonomique et interprétative. Puis, nous analysons les pratiques de conception qui participent à produire l'illusion d'une continuité entre le corps et l'écran. Enfin, il s'agit de montrer que, alors que les écrans dépassent largement la frontière du doigt pour produire de la donnée à partir du corps entier, ses discours et ses imaginaires contribuent à faire diversion des enjeux économiques et de pouvoir qui les traversent.

\begin{abstract}
This article proposes to denaturalize the notion of "tactile" and the metaphorical vocabulary that accompanies it - such as "fluidity", "interactivity" or "responsiveness" - which engage a fascinated relationship to the contemporary screen. We are interested in the touch of the screen, that is to say the moment when the user manipulates the interface with his fingertip: it is in this meeting between the technical and the corporeal that the continuity between the body and the screen is played. The aim is to observe the ways in which this finger is digitized, treated by the screen; to question the tactile as a technical, computer process; as a human, semiotic and anthropological process; and as an economic process, typical of a new industry. In a first step, we will return to the conditions of elaboration of the critique of the notion of "tactile" and the conceptual tooling that it requires, that of writing. We propose to reinscribe the screen first in its technical, ergonomic and interpretative dimension. Then, we analyze the practices of conception which participate to produce the illusion of a continuity between the body and the screen. Finally, it is a question of showing that, whereas the screens largely exceed the finger border to produce data starting from the whole body, its speeches and its imaginary contribute to make diversion of the economic stakes and of power which cross them.
\end{abstract}




\section{Introduction : du « coup de doigt » sur l'écran}

Réactif à la pression du doigt ou à son effleurement, l'écran contemporain s'est vu attribuer un adjectif, celui de « tactile». Sinon mettre l'accent sur l'absence de médiation technique pour son usage, d'un clavier, d'une souris ou d'un stylet, celui-ci ne dit rien de l'écran. Ce terme, qui dote un objet inanimé de la reconnaissance d'un attribut humain, le toucher, rend opaque le fonctionnement de ces objets si ordinaires. Il y a en plus, dans la dénomination « tactile », une normalisation de la gestuelle pour manipuler l'écran qui laisse penser que les gestes pour se servir du numérique sont neutres dans l'activité, réellement insignifiants et vont d'eux-mêmes. Il s'est pourtant popularisé avec le développement et l'adoption massive des dispositifs mobiles tels que les smartphones ou les tablettes, manipulables du bout du doigt, si bien qu' « écran tactile » est désormais un syntagme figé. Notre propos vise sa dénaturalisation.

Pour cela, nous allons interroger ce qu'il se passe dans la manipulation des écrans des dispositifs mobiles, ce que « tactile » recouvre en termes de processus et d'enjeux. De ce point de vue, le coup de doigt sur l'écran est un moment crucial : c'est lorsque l'utilisateur l'effleure - du pouce ou de l'index - que se manifeste la sensibilité de l'écran, répondant aux doigts de l'usager. Dans ce contact furtif se condenserait l'embrayage du tactile, c'est-à-dire la mise en mouvement de l'écran et l'impression de le traverser tout en l'effleurant. En proposant une optique sur l'extrémité du membre, il y a une possibilité de bascule vers des échelles plus larges : il s'agit de regarder la façon dont on les manipule pour comprendre comment cette activité condense un grand nombre d'enjeux. S'intéresser au « tactile », c'est donc se demander comment le doigt de l'usager est numérisé, ou encore, quels sont les procédés sémio-techniques permettant la digitalisation du doigt par les écrans.

In fine, la perspective est la critique de la notion de "prothèse ». On se trouverait, avec ces écrans, à un moment de transformations. Quelque chose se jouerait, dans la manipulation de l'interface, de l'ordre de l'anthropologique, si bien que, selon certains discours, ils marqueraient une rupture dans les rapports du corps à la technique. Les écrans passent pour être une technologie intime, intégrée et incorporée : on ne pourrait plus s'en passer. Initiée par Marshall McLuhan (1964), la notion traverse les disciplines académiques et les recherches sur le numérique, comme les discours fantasmant une technique dans le prolongement l'être humain. Ce n'est pas l'angle de l'hybridation, mais celui de la relation entre l'usager et le dispositif qui nous intéresse. Plutôt que de conjecturer sur le devenir prothétique des machines, c'est le rapport entre l'être humain, l'écran et les dispositifs ${ }^{1}$, tel qu'il est et tel qu'il s'actualise, que nous souhaitons observer. Dans la rencontre entre du corporel et de l'inorganique - le geste à la surface de l'écran - se jouerait une continuité avec le corps. C'est à l'articulation des deux qu'il convient de situer le regard pour comprendre comment se joue ce qui passe pour un prolongement des facultés humaines par les technologies mobiles et tactiles.

L'objectif de cette contribution est de démystifier le tactile et de montrer que c'est une écriture industrielle iconique de pratiques généralisées. Pour cela, nous reviendrons d'abord sur les enjeux de la dénaturalisation du terme tactile et soulignerons la nécessité de le prendre comme un phénomène. La notion d' " écriture » permet alors de se donner un concept pour le comprendre et les moyens de voir « sous » et autour de l'écran. Ensuite, en faisant varier les approches et les échelles, nous décrirons les différents processus techniques et humains impliqués dans la rencontre entre le doigt et la surface vitrée. Cela permettra de comprendre la façon dont ces objets donnés à manipuler impliquent la sensorialité. Nous reviendrons également sur les usages sociaux de la notion de « tactile». De tout cela, il ressort une représentation de la corporalité qui justifie que l'on parle de "viande» humaine plutôt que de "chair». Nous contextualiserons enfin cette activité de manipulation des écrans dans ses cadres économiques en vue de comprendre l'encodage du geste dans les couches calculatoires de la machine et affirmer que c'est l'idée même du «tactile » qui doit changer.

\section{Ce que le « tactile " fait à l'écran}

Dans sa Contribution aux avancées de la connaissance en Information-Communication, Bernard Miège s'oppose aux notions qui, en SIC notamment, empêchent le questionnement scientifique et imposent une vision déterministe des recherches et des champs concernés : les « notions-écrans » (Miège, $2015: 222$ ). Sorti du champ de l'épistémologie, ce concept s'applique à la notion de «tactile» qui circule dans le social : le « tactile» fait écran. Nous proposons alors de remettre en question le vocabulaire métaphorique de rendu de l'interface pour réinscrire l'écran dans ses pratiques et ses usages, tout en considérant ses modes de conception et d'élaboration. C'est que, l'écran tactile est un phénomène humain construit en amont par des acteurs ayant certaines manières de faire, que la notion d'écriture permet de saisir finement.

\subsection{Interroger le vocabulaire métaphorique de rendu de l'interface}

L'écran dit tactile s'accompagne d'un vocabulaire, à l'origine spécialisé en design d'interface, désormais passé dans la langue vernaculaire : il est qualifié de «fluide», quand il répond agréablement aux injonctions gestuelles de l'usager ; d'《 interactif », quand il répond à ses commandes ; ou encore de « réactif » quand il est particulièrement « sensible » à

\footnotetext{
${ }^{1}$ On entend ici par dispositif le sens commun du terme, à savoir : « Ensemble d'éléments agencés en vue d'un but précis » (Centre National de Ressources Textuelles et Lexicales, entrée « dispositif»)
} 
la manipulation. Il passe aussi pour être "frictionless", "sans friction", ou encore sans accrochage dans sa manipulation. Métaphores ou néologismes, ces termes ne permettent pas de rendre compte et de comprendre le fonctionnement de l'écran, ils le rendent fuyant et insaisissable : l'écran serait liquide, aurait du répondant ou encore une sensibilité à un sens anthropologique, et tout cela à la fois. Les mots ne parviennent pas à décrire ce qui a lieu dans la manipulation, tout en buttant sur les images qu'ils évoquent. Il revient au chercheur d'en montrer la limite et de les contourner.

Dans un texte paru en 1993, Hélène Godinet fait l'examen du vocabulaire de l'informatique et des terminologies employées pour en nommer les objets. Elle s'interroge sur l'intérêt de ce type de jargon pour la recherche scientifique et revient pour cela sur la genèse du néologisme ${ }^{2}$. Elle constate que le néologisme, qui relève d'une démarche pragmatique, du bon sens, s'impose du fait de son efficacité : il se fixerait car il n'engagerait pas de confusion quant à l'objet auquel on a affaire. Autrement dit, les termes choisis pour en parler dépendent des perceptions que le grand public en a. Elle prend même l'exemple de l'écran tactile : « Nul besoin de dictionnaire pour adopter le 'crayon optique' ou 'l'écran tactile' » (Godinet, 1993). «Tactile» se serait imposé parce que le terme est «opératoire, fonctionnel» (Ibid.), c'est-à-dire qu'il fait surgir à son évocation un ensemble de pratiques et de possibilités, constitue à lui seul un mode d'emploi. Le terme souligne qu'il n'y a pas besoin de médiation, de périphérique supplémentaire pour l'usage de l'écran, mais pour autant, ne dit pas comment il fonctionne. Polysémique, le terme « tactile », accompagné de ces divers adjectifs métaphoriques, rend dès lors compte d'une double caractéristique. À savoir que l'écran serait en quelque sorte «palpable» (synonyme donné par le Centre National de Ressources Textuelles et Lexicales, entrée " tactile»), « triturable » du bout des doigts; et aussi, qu'il serait sensible à l'activité humaine sur sa surface, réactif au toucher. Mais il se révèle assez approximatif : ce que l'utilisateur «tripote », c'est une surface lisse, en verre, ni malléable, ni variée dans son toucher ${ }^{3}$. Il s'agit d'un rendu de l'interface, et non de l'interface dans son essence et dans son fonctionnement dynamique.

L'enjeu est également politique. Godinet met au jour les rapports de pouvoir traversant ces néologismes qui, selon elle, manifestent la relation des usagers aux dispositifs informatiques. Elle affirme ${ }^{4}$ ainsi que certains termes néologiques témoignent d'une emprise de l'objet sur ses usagers. S'en défaire est primordial pour les saisir scientifiquement, mais aussi car ils entretiennent cette confusion. Le terme « tactile » s'inscrit dans cette tendance. Prêter à l'écran des qualités qu'il n'a pas, et qui sont, de surcroît, liés à des attributs humains, tend à le magnifier ; dire qu'il est "tactile», « réactif », « fluide », c'est lui accorder des facultés et des vertus, presque malgré soi, et s'enfermer dans un ravissement de l'écran. Cette démarche de requalification et de reconceptualisation de l'écran vise finalement à dénaturaliser les rapports avec les médias informatisés, comme avec les industries culturelles et marchandes du numérique.

Pour ces raisons, épistémologiques et politiques, il n'est pas pertinent de se contenter des termes usuels pour l'étude des écrans contemporains : tactile, fluidité, interactivité, sont des notions qui président à la réception, teintent les pratiques et cela est perceptible dans les discours des usagers qui se les approprient. Relever ces représentions sociales est essentiel pour comprendre la grille d'interprétation et certaines logiques sous-jacentes des usages, et engage à déplacer la question. Il faut s'outiller de concepts, non pas interroger l'interface comme objet, mais le rendu de l'interface comme construction techno-sémiotique et discursive. Pour contourner ce vocabulaire métaphorique, nous proposons de le faire monter en phénomène : c'est-à-dire de le considérer dans ses apparitions et manifestations au sens ou à la conscience (Centre nationales de Ressources Textuelles et Lexicales, entrée " phénomène »). Le " tactile » dans sa version écranique est un objet, mais aussi un phénomène observable, du phénoménal, un objet que l'expérience connaît, mais qui échappe à la conceptualisation.

\subsection{L'écran tactile, un phénomène anthropologique construit}

Nous proposons d'aborder la manipulation de l'écran comme un phénomène anthropologique et d'en étudier les conditions d'élaboration : il est construit, préparé et encadré en amont.

D'une part, la manipulation tactile engage des processus corporels, cognitifs, interprétatifs, propres à l'être humain. Ce moment est particulier : il implique le corps, ses sens et un processus de sémiose, d'interprétation de la situation et de la performance à l'écran, de ce qui se joue à sa surface. D'autre part, les interfaces dites tactiles sont le fruit d'un travail créatif, de développement, de mise en production et de commercialisation-qu'il s'agisse de l'écran comme des services qu'il médiatise. Ce que l'usager tient entre ses mains est le résultat d'un travail long de construction et tout ce qu'il offre à la manipulation a été testé, "bêta-testé », potentiellement déconstruit et repensé en fonction des résultats de navigation obtenus, restitués aux concepteurs, designers et développeurs, sous forme de données ${ }^{6}$. Le "phénomène

\footnotetext{
${ }^{2}$ Elle en définit l'origine non au moment de l'élaboration, mais de la circulation de l'objet : ce serait l'usager qui, en général proposerait le(s) terme(s), d'après son expérience, pour le nommer.

${ }^{3}$ Certaines interfaces ont cependant un système de vibration attestant de la réalisation d'une action.

4 «L'emploi inconsidéré de ces néologismes révèle une sorte de fascination devant la puissance présupposée de l'outil. Il trahit une certaine confusion entre la vie mentale de l'individu et les potentialités accordées à l'ordinateur » (Godinet, Ibid.) ».

${ }^{5} \mathrm{Ou}$ testé selon deux propositions pour déterminer la plus pertinente pour l'usager.

${ }^{6} \mathrm{D}$ 'après les propos recueillis auprès d'un designer d'expérience, octobre 2020.
} 
tactile » repose sur un construit, résulte de manières de faire, de classes d'acteurs spécialisés. Il est pensé par les designers, résulte de préceptes et de manières de concevoir l'expérience de l'usager et de normes définies.

Pour saisir le « phénomène tactile », nous proposons ce point de bascule entre l'usager et la technologie, la médiation gestuelle qui en permet la manipulation. L'adjectif lui est d'ailleurs étendu : on parle de «gestes tactiles » et plus souvent encore de "gestualité fluide ». C'est précisément là que se joue le tactile comme phénomène. D'un côté, cela permet de mettre au jour les processus humains impliqués dans la manipulation et son interprétation. D'un autre côté, ils permettent d'appréhender le tactile dans sa dimension créative et industrielle permettant d'interroger les conditions d'élaboration et de production de ces gestes d'appui ou de glissé sur l'écran au moment de la création de l'interface. Ils autorisent des effets de basculement du niveau social et culturel, au niveau technique et économique. C'est, en somme, le «tactile » comme spectacle et ses logiques de fonctionnement qui est le sujet du questionnement. Sa déconstruction demande de confronter des analyses sémiotiques de l'écran, avec des discours du design et les logiques économiques qui les sous-tendent. Pour cela, nous nous sommes appuyée sur un corpus d'applications usuelles en vue d'analyser des interfaces d'écrans tactiles dans des contextes et des applications. Nous avons articulé des analyses sémiotiques, sémiodiscursives et pragmatiques aux discours de bonnes pratiques, aux manières de faire et aux routines du design afin de comprendre comment et pourquoi cela passe pour du «tactile». Mais aussi, parce que l'écran est pris dans des enjeux stratégiques et économiques, il est indispensable de le situer dans son contexte industriel, de replacer cette technique dans son contexte économique et dans les services qu'il outille.

La démarche est communicationnelle, par son approche, et compréhensive, par sa méthode. Pour changer de regard sur le « tactile », il n'est pas possible de le prendre en dehors de ses performances et de ses actualisations.

\subsection{Une approche de l'écran et du geste par l'écriture}

Pour réinscrire la manipulation de l'écran dans ses logiques sociales, professionnelles et économiques, l'étude des écrits d'écran (Souchier, 1996) est heuristique. Son tour de force est d'avoir remis l'écriture au cœur des techniques modernes. Elle propose un concept pour s'emparer des gestes, l'écriture, mais aussi, à travers sa proposition de « couches », une façon de déconstruire les « épaisseurs » de l'écran :

«[...] ce que l'écrit a perdu en épaisseur tangible, en dimension volumique présente dans l'objet imprimé, il l'a recréé par ailleurs dans une organisation logique qui se présente comme une superposition de couches. Du niveau le plus profond où se calcule la représentation codée des caractères alphabétiques, au niveau le plus superficiel où se manifeste à l'écran la mise en forme des médias informatisés, s'élabore un dispositif complexe de construction, d'accès et de représentation des textes et documents ». (Cotte, 2004).

Dans cette perspective, on peut découper le geste en plusieurs actions synchrones : c'est une activité corporelle articulée à une couche sémiotique, la représentation de quelque chose de lu et d'interprété par l'usager ; articulée à une action dans l'architexte ${ }^{7}$, dans la couche architextuelle, celle du logiciel lié à l'interface que l'utilisateur peut activer; à une couche calculable, celle du programme et de ses algorithmes. S'intéresser aux gestes de manipulation comme des outils de cette écriture numérique permet d'intégrer cette triple dimension (sémiotique, corporelle et algorithmique).

L'étude des écrits d'écran élargit en outre la notion de geste: c'est un geste d'écriture et un acte de lecture. L'utilisateur, au moment d'effectuer un geste donné, interprète la situation. Il lit, déchiffre, ce qui lui est proposé à l'écran. Cela conduit à interroger les processus de construction du sens, de sa valeur, et des interprétations diverses. Ce sont des gestes-textes, à lire et à interpréter. Enfin, l'étude des écrits d'écran impose de s'intéresser aux modes de fonctionnement algorithmiques et économiques de numérique. Dans cette perspective, la manipulation permet la captation de données et s'inscrit dans les couches du fonctionnement logiciel et algorithmique. Considérant l'algorithme comme de l'écriture (Cotte, ibid. ; Souchier et al., 2019), c'est du texte qui est produit par ces gestes. On peut repérer un ancrage et un rôle économique : ils participent de la captation des données et du fonctionnement général de ces dispositifs.

En considérant la manipulation comme un geste de lettrure (Souchier, 2012a), combinant la lecture et l'écriture, on se donne la possibilité d'inclure le logiciel et la couche calculatoire de ces écrans, le contexte économique et industriel. L'approche par l'étude des écrits d'écran permet de les contextualiser et de tenir compte des enjeux de captation et de valorisation. Ce questionnement s'inscrit finalement dans ceux des rapports de l'humain au numérique et au monde. Il faut les prendre comme des gestes du rapport à la technique, à soi et à ses représentations.

\footnotetext{
${ }^{7}$ Ici, on s'appuie sur la définition donnée par Yves Jeanneret de la notion d'architexte comme : « outil présent dans les écrits d'écran qui se situe à l'origine des actes d'écriture et de lecture des usagers et en régit le format, si bien que notre propre écriture est conditionnée par l'existence de cette écriture en amont de la nôtre » (Jeanneret, Lexique, entrée « architexte »)
} 


\section{Des écrans sensibles à la viande humaine}

Pour dénaturaliser le tactile, c'est-à-dire mettre à distance les discours autour de sa « réactivité » et les représentations cognitives que l'on en a, nous proposons de le poser sur le plan de son fonctionnement technique, c'est-à-dire de partir de ses propriétés matérielles. Observer l'écran par le prisme de la programmatique, de l'ergonomie et de l'interprétation permet d'oser des pistes d'analyse et de compréhension.

\subsection{Le digital, une modalité d'interaction chargée de sens humain}

Comment se défaire de l'idée que l'écran serait tactile, réactif au toucher humain ? Pour cela une œuvre proposée par quatre étudiants en art, dans le cadre d'un projet universitaire, est intéressante. Il s'agit de « Tender, it's how people meat $»^{8}$, qui fait une critique de la gestion des personnes par l'application de rencontre Tinder : l'usager serait réduit à n'être qu'un morceau de viande. On retrouve cette métaphore dans les imaginaires critiques usuels de l'application (Garmon, 2016). Les artistes ont créé un dispositif mettant en scène, de façon littérale, ce constat. Attaché à un bras mécanique, un steak cru est actionné en boucle pour valider tous les profils (tout cela permet d'optimiser l'algorithme). Ce dispositif nous intéresse surtout car il montre que l'écran n'est sensible à rien du tout, sinon, à la viande.

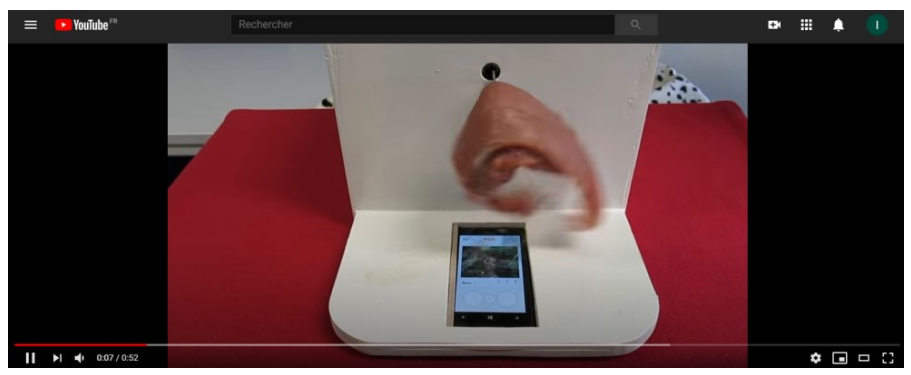

Figure 1. «Tender, it’s how people meat ». Source : https://www.youtube.com/watch?v=9EPXVN5IMhQ - consulté le 11/11/2020.

Pour le dire plus finement, l'écran est réactif à la peau de l'usager, à ses propriétés et ce sont ses qualités corporelles, ou ce qui leur ressemble, qui le font agir. Comme ici, où l'on suppose qu'un steak cru doit posséder des propriétés physiques proches de celle du doigt de l'être humain. Il y plusieurs types d'écrans répondant plus ou moins aux commandes des usagers, selon qu'ils sont dotés ou non de la technologie multitouch. Les écrans des dispositifs mobiles le sont quasiment tous et sont dits « tactiles capacitifs ${ }^{9}$. En fait, la présence d'un ou de plusieurs doigts sur des points précis produit une perturbation électrique, celle-ci est traitée par une grille sous la surface qui les convertit en coordonnées ensuite renvoyées au système d'exploitation. C'est une double opération de traduction qui s'opère, du toucher en signal électrique en coordonnées. L'écran « capacitif» est en verre, " recouvert d'un conducteur transparent, généralement à base d'oxyde d'indium étain $»^{10}$ et équipé de capteurs à ses coins destinés à mesurer les variations de charge électrostatique. Il est construit pour réagir à la peau de l'usager, et à ses caractéristiques plus ou moins spécifiques. Ce qui est mesuré, c'est la perturbation induite par le doigt posé sur la surface : ce n'est pas la peau qui est détectée, mais les « coordonnées du toucher ». Ce contact peut être digital, passer par un stylet, de la poussière ou même du gras. Cette incapacité de distinction de l'écran est notoire quand le tactile ne réagit pas au contact d'un gant, ou s'active malheureusement parce qu'il pleut. Dans ces moments frustrants, la pensée magique se heurte à une surface en verre. Ce type de mésaventure de l'écran illustre bien la trivialité - au sens commun - de l'écran.

Sur le plan technique, les écrans sont sensibles à la viande tout court, bien qu'une simple viande erratique ne les agira pas adéquatement. Cela est visible dans la vidéo de ce steak activé en boucle où la manipulation fonctionne car c'est toujours la même action, un glissé vertical, qui est faite, alors que le «tactile » attend un projet conscient. C'est-à-dire que pour une manipulation de l'écran plus complexe (écrire un message, envoyer une photo, faire défiler un fil d'actualité, partager un contenu...), il faut engager une action précise : le « digital» s'est imposé comme modalité de manipulation chargée de sens humain. Pour comprendre cela, nous proposons un autre spectacle qui circule sur la plateforme YouTube: il s'agit de vidéos montrant des singes manipuler des téléphones ou des tablettes. Si les singes sont dotés de mains comparables anatomiquement à celle de l'être humain, qui sont bien détectées à la surface de

${ }^{8}$ https://www.vice.com/en/article/yp5bew/this-piece-of-meat-just-swiped-right - consulté le 29/10/2020.

${ }^{9}$ D'après la documentation informatique trouvée en ligne, «La technologie d'écrans tactiles capacitifs s'appuie sur une surface solide de type verre, laquelle est parcourue par une grille électriquement chargée et presque invisible à l'œil nu. L'accumulation de charges électriques sur la plaque de verre lors du contact des doigts de l'utilisateur transfère une partie de ces charges dans les doigts, ce qui provoque un déficit qu'il suffit ensuite de localiser pour traiter l'information. Le traitement de l'information se fait au moyen d'un algorithme de calcul intégré directement dans le périphérique. Il détermine le ou les points d'impact (dans le cas du multi touch), le sens du mouvement, parfois la pression exercée, la vitesse, et agit en conséquence» http://interfacetactile.com/ecran-tactile-capacitif - consulté le 29/10/2020.

${ }^{10} \mathrm{https}$ ://www.futura-sciences.com/tech/definitions/technologie-ecran-tactile-539/ - consulté le 29/10/2020. 
l'écran, ceux-ci par exemple ne «manipulent» pas : il n'y a pas interprétation de l'activité comme le ferait un usager face à son écran. Il y a une performance dans l'action « digitale» qui n'est pas présente dans la manipulation de l'écran par des singes : leurs mains collent au «tactile» a priori, mais il n'y a sans doute pas chez eux d'interprétation et d'intention comme dans nos manières d'agir. C'est pour cela que, lorsqu'on voit un singe tapoter l'écran, l'idée de la prothèse est en décalage et fait sourire. Alors que la manipulation telle que la pratique un singe relève d'une activité mécanique, celle de l'humain implique une dynamique sensorielle et interprétative spécifique, de déchiffrement de l'écran. Cela justifie que l'on parle d'écriture, et que l'on cherche à faire varier ces termes ordinaires. Ce spectacle confirme la nécessité d'un concept opératoire pour parler de ces gestualités en rendant obsolète le paradigme de la manipulation tactile : nous avons proposé (2017) celui de « digipulation ».

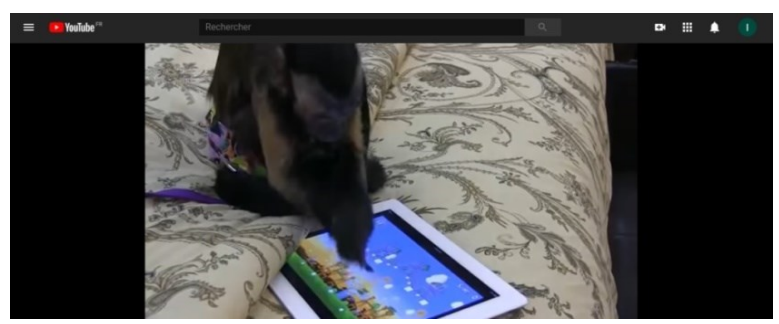

Figure 2. Vidéo présentant un singe manipulant un IPAD. Source : https://www.youtube.com/watch?v=EbBNliFEPbw - consulté le $11 / 11 / 2020$.

\subsection{Un phénomène qui passe par une technicisation de la main}

Par conséquent, il faut entendre par «tactile», la rencontre entre un humain avec un doigt capable de pression, de précision, de toucher et un écran techniquement abouti pour réagir à son contact. Cet écran technique est conçu pour épouser, se fondre dans la main. Par exemple, la taille des écrans est pensée pour qu'ils soient aisément tenus, dans la paume ou à deux mains (et les programmes suivent les basculements des écrans). Ou encore, leur finesse cherche à les rendre aussi légers, qu'agréables à tenir par la disparition des résistances au doigt. Outre leurs caractéristiques techniques, les écrans tirent parti des propriétés humaines sur le plan anatomique.

L'ossature de la main laisse, pour commencer, entrevoir de très nombreuses possibilités pour la manipulation délicate d'objets :

«L'articulation du doigt en plusieurs phalanges et la capacité du pouce à s'opposer à chaque doigt lui donnent une possibilité de prises multiples depuis celle, en force, où elle se referme sur l'objet à la manière d'un crampon, à celle où la précision et la finesse l'emportent. La structure ostéomusculaire favorise la motricité, la mobilité, la souplesse, la puissance, l'habileté pour des tâches innombrables impliquant la précision ou la force. Dans les techniques du corps, la main, avec l'affinement que lui procurent l'œil et l'intelligence pratique, est une pièce maîtresse par ses possibilités d'apprentissage, de métamorphose, d'adaptation aux circonstances ». (Le Breton, 2006 : 186)

Pour les concepteurs, il s'agit de rendre optimale la relation entre l'écran et le corps, mais aussi de tirer parti des potentialités de ce dernier. Les gestes ensuite peuvent être réalisés par la majorité des usagers, avec des «boutons » conçus pour être accessibles à toutes tailles de doigts (il est également possible d'ajuster la taille du contenu de l'écran et des boutons). Les écrans ensuite exploitent l'articulation, la mobilité du pouce, en mesure d'appuyer sur le bouton central en bas de l'écran (bouton principal de beaucoup de smartphones) et d'atteindre presque toute la surface de l'écran. La finesse et la dextérité de l'action permise par la main humaine permet donc une certaine variabilité d'actions. On peut dire qu'il y a maximisation, par les concepteurs de ces écrans, de la main, de sa " polyvalence », de ce que Leroi-Gourhan a appelé sa « libération » (1964). Cela produit une technicisation et un changement de statut de la main dans le monde contemporain et s'accompagne de sa valorisation par la conception et par le social. La main et les doigts sont pris en compte en amont, par les ergonomes et concepteurs qui s'appuient sur leur fonctionnement et les ont institués en «modèle ». Sur les tableaux panoramiques des gestes, tels que le Touch Gesture Guide (Figure 3) la main est l'archétype, l'étalon, le squelette de l'écran. 


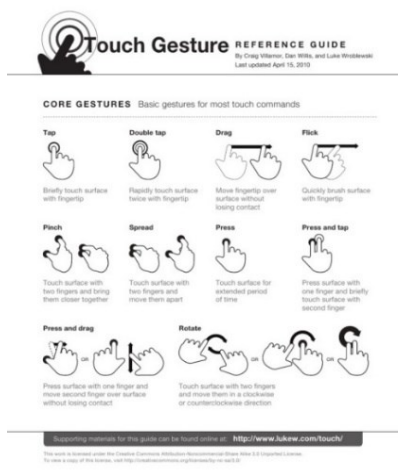

Figure 3. Extrait du Touch Gesture Reference Guide, guide de référence des gestes de manipulation des interfaces. Source : https://www.slideshare.net/pierrre/touch-gesture-guide - consulté le 11/11/2020.

La main se voit finalement octroyer plusieurs statuts : ceux d'outil, de modèle et d'organe en changement. Dans Les Techniques du Corps, Mauss propose le concept de «technicisation » pour décrire la transformation du corps en outil. C'est ce qui permettrait à la technique d'avoir prise sur le monde, d'agir sur lui :

« Sans cette technicisation, c'est-à-dire sans insertion active des corps dans le système des normes techniques vues comme source et ressources de l'activité individuelle et collective, aucune technique ne pourrait avoir prise sur le monde ». (Ouvrier-Bonnaz, 2011).

Les dispositifs mobiles et tactiles font de la main une ressource pour l'activité et c'est précisément le bout du doigt qui est impliqué. La «pulpe » de l'index ou du pouce passe, avec ces écrans, pour un outil de préhension et d'action sur le monde.

Cette technicisation s'accompagne également de discours autour de la main, comme pour en soutenir et en attester la puissance. Le doigt est valorisé dans les représentations du numérique. Il semble être le cœur du pouvoir de l'usager : la représentation du numérique passe souvent par une focalisation sur le doigt, comme symbole de ses potentialités - par exemple, la recherche du terme « numérique » sur Google Images donne à voir une sélection d'images mettant la main et le doigt en centralité. Enfin, « conséquence de ces nouveaux usages, on prévoit déjà des modifications importantes dans les muscles de la main» (Leader, 2017 : 12). Outre l'importance accordée aux écrans dans le développement anatomique, avec l'idée sous-jacente que la technique forme, ou plutôt déforme le corps, ce type de discours spécialisés et sociaux manifestent une conscience diffuse des changements dans l'usage des mains et de ses sollicitations.

Revenir sur les façons dont se détermine le contact entre les opérations techniques, informatiques et sémiotiques permet de déplacer la question : d'une part, certaines propriétés de la peau humaine et des doigts sont efficaces dans le modèle ergonomique de l'écran; d'autre part, c'est un esprit humain, avec ses intentionnalités et ses modalités interprétatives qui composent le « tactile». Il n'y a pas d'écran « tactile», il y a des doigts qui s'agitent. D'où vient alors l'impression tenace de sensibilité, d'une continuité entre le doigt et l'écran?

\section{Une illusion de continuité entretenue par la conception}

Passons au niveau sémiotique de l'écran. S'il n'y a pas de «tactile », il y a bien l'impression, pour l'usager, d'une continuité entre sa pensée, son geste et l'écran. En cela, les sensorialités humaines sont le jeu d'une illusion cognitive. Il y a d'un côté, un travail technique pour construire l'interface la plus «fluide» possible; et d'un autre, une transformation lente des attributs supposés du « tactile», de ses attentes et de ses modes d'appréhension qui soutiennent cette impression de continuité entre le geste et l'écran.

\subsection{Une illusion cognitive soutenue par un travail d'institutionnalisation du signal en signe}

On peut décomposer le geste de manipulation de l'interface en deux processus synchroniques. Au moment où le doigt touche l'écran, il met en marche deux activités antagonistes et complémentaires : une activité de toucher et une autre, sémiotique, traitant les signes à l'écran. C'est une activité d'analyse du geste en même temps qu'il est réalisé. L'utilisateur a certains de ses sens, à l'instar du toucher et de la vue, ou encore l'ouïe, qui sont sollicités par ces écrans. Leur sollicitation asynchrone engage une illusion cognitive puissante, soutenue par les manières qu'ont les concepteurs de construire l'écran.

La manipulation d'un écran engage une activité de lecture et d'interprétation pour l'usager : la compréhension de ce qui se passe à l'écran passe par l'analyse des signes, des formes, des procédés et des propositions. Mais surtout, lors de la 
manipulation, l'usager voit l'écran réagir au contact de son doigt, suivre tous ses mouvements. Il y aurait, de façon simultanée, une activité " basse » de toucher (des doigts sur l'écran, à la surface d'un écran en verre) et une " haute », sémiotique et cognitive, de lecture et d'interprétation de l'écran ${ }^{11}$. C'est la mise en relation de ces deux activités qui donne l'impression d'une continuité et d'une sensibilité de l'interface au doigt de l'usager ${ }^{12}$. Cette impression de sensibilité de l'interface reposerait sur la nature "synesthésique " de la perception ${ }^{13}$. C'est-à-dire que, faisant agir un écran, l'usager ne décompose pas son action, il en opère une interprétation immédiate. L'effort d'interprétation en plus est tourné vers les propositions d'activité (partager un contenu, commenter, consommer etc.) et moins vers le décodage du fonctionnement de l'écran, accepté depuis longtemps et infra-ordinaire ${ }^{14}$. On peut parler d' «illusion cognitive», illusion qu'il y aurait continuité entre la main et l'écran ${ }^{15}$. Il y a une confusion : ce qui est perçu prend le pas sur ce que pourrait élaborer l'usager, une déconstruction de l'ensemble. Cette illusion cognitive repose sur la nécessité de donner sens à ce que l'on fait, d'en produire une interprétation. Contrairement à la prétention de l'adjectif tactile de l'absence de médiation, le doigt est finalement une médiation technique traduite, par l'activité sémiotique et sensorielle, en une sensibilité de l'interface.

Cette médiation technique, et son rendu, font l'objet d'un travail minutieux par le développement : pour que cela ait l'air sensible, que le tactile " prenne », le rapport doigt / écran doit être parfaitement élaboré. Cela met en évidence le travail central du designer d'interface : c'est sur le développement de l'interface, de l'écran et de ce qu'il propose à la manipulation, que l'expérience «tactile» s'établit. Si on considère son fonctionnement technique, il repose sur la détection d'un signal (émis par le contact du doigt) selon les coordonnées, la vitesse, le trajet, effectués par l'objet ou le membre à sa surface et transformés en signe pour la machine. Il y a ensuite retraduction pour l'usager à l'écran que l'action a été enregistrée (passant par un changement d'état de l'écran ou d'une forme spécifique). Cela indique que le tactile repose sur un travail du designer d'interface pour transformer adéquatement le signal en signe. Il faut pour cela que, en amont, ait été correctement programmée l'action, soit la reconnaissance du bon geste au bon endroit, et sa traduction immédiate, quasi instantanée à l'écran. C'est cette reconnaissance par l'usager, de son geste et de son programme d'action souhaité et rendu à l'écran, qui donne l'impression d'une continuité entre l'esprit et ce même écran. Cette synchronisation immédiate et l'opérativité du geste donnent l'impression évoquée d'une continuité entre la pensée de l'usager (son projet), son geste et l'écran. Tout se passe dans les changements visibles de l'écran et repose sur un travail sémiotique précis des concepteurs des interfaces numériques. Ceux-ci participent d'une institution entre le signal (le touché) et le signe à l'écran (le rendu de l'interface). Alors que le geste discrétise l'énonciation de l'usager - il la découpe en une action donnée -, le design vise à réconcilier le geste et son action. De ce point de vue, c'est bien une affaire de technicité et de précision du développement, et non de magie ou de sensibilité. L'interprétation, par l'usager, que son activité de manipulation est effectivement « tactile» passe par une finesse du design pour lier la manipulation de l'écran et ce qu'il renvoie à l'usager.

Parler de «tactile », comme de « réactivité » et de «fluidité », empêche de voir à quel point ces phénomènes sont construits à un niveau technique par des acteurs ayant certaines conceptions. C'est à ce niveau technique que se construit un rapport propre entre le geste correct et son interprétation. Ainsi, lorsque l'on actualise un écran, on retrouve les conceptions des concepteurs, largement structurantes, qui eux-mêmes s'inspirent de gestes d'écran comme de gestes qu'ils connaissent. En ce sens, les interfaces ne sont pas tactiles et intuitives mais sémio-techniques et mimétiques.

\subsection{Un terme qui s'est chargé de valeurs}

Les manières de développer les interfaces construisent l'impression d'un écran en continuité de l'usager, qui réagit à son toucher. En même temps que ces tours sémio-techniques, l'imaginaire, la perception, et les enjeux du « tactile» se sont transformés. Cette pensée de l'écran masque cependant l'objectivation du corps même de l'usager, si bien que parler de « viande » semble s'imposer.

La pensée d'un écran qui s'active au contact du doigt de l'usager, qui répond finement à son geste à sa surface, ne s'est pas immédiatement imposée. L'idée de l'écran en effet a évolué au fil des innovations qui l'ont traversé. Il y a surement

11 Platon, dans le Phédon, défend l'idée d'une « bassesse » de la perception sensible et il estime impure la connaissance autrement que par le raisonnement: «À travers les yeux de l'âme et de la pensée, l'homme pénètre les arcanes du sensible, il écarte les tentures qui condamnent la réalité du monde, et il accède à une intelligence purifiée du sensible » (Le Breton, 2006 : 60). Il oppose perception et cognition, interprétation et raisonnement.

12 Par exemple, face à un écran tactile, si l'utilisateur fait glisser son doigt sur l'écran d'accueil et que tous les menus bougent de manière latérale en suivant son doigt, il y a analyse, sans conscientisation, que l'activité est prolongée «par l'écran »; il « comprend » qu'il fait défiler les différentes pages d'écran. L'inverse a déjà été, par tous, expérimenté : le tactile échoue quand l'écran ne répond pas parfaitement aux commandes de l'usager et à ses gestes sur sa surface ; qu'il y a un décalage entre le geste et ce que lui renvoie l'interface.

${ }^{13}$ Rappelons que « la perception synesthésique n'est pas un ensemble de données, mais une prise globale du monde sollicitant à tout instant l'ensemble des sens » (Merleau-Ponty, 1945: 265 cité par Le Breton, $2006: 56$ ).

14 Souchier (2012) définit ainsi la notion à partir des travaux de George Pérec, telle que : « de petites choses, [...] des détails infimes qui nous fondent et qui, à force de petitesse, disparaissent à nos yeux inattentifs » (Ibid.).

${ }^{15}$ C'est un paradoxe : on sait que l'écran n'est pas en continuité avec le doigt, toutefois, on ne peut s'empêcher de le percevoir comme un peu magique, dans notre prolongement. 
eu un moment de basculement où l'expression «toucher l'écran» est passé de l'incongruité au pléonasme. Ce basculement s'est accompagné d'un changement de perception et d'une attente particulière de l'interface de la part des usagers. Au moment des premiers écrans tactiles accessibles au grand public, la consigne «Toucher l'écran» accompagnait l'appropriation du dispositif. Petit à petit, cette instruction a disparu. Cette disparition indique une naturalisation de l'usage : il n'est plus nécessaire d'avoir comme consigne qu'il faut " toucher » l'écran, l'usager y est habitué. Cette habitude, ou ordinarisation de la pratique, s'est accompagnée d'un changement de posture pour l'usager : de passif devant un écran de type télévision ou cinéma, il est devenu actif avec $1^{\text {'ordinateur }}{ }^{16}$, une intervention sur l'écran est désormais attendue de lui. La naturalisation de l'écran " tactile » a nécessité un changement de posture de l'usager, inclut une pragmatique de l'objet, son engagement et son basculement dans l'action : c'est l'« effet de l'interface » introduit par Alexander Galloway (2012) : " Avec l'ordinateur, il ne s'agit plus, selon [lui], de voir ou de croire en ce que l'on nous montre mais plutôt d'agir à l'intérieur d'un environnement complexe. » (Treleani, 2014). En même temps, une attente s'est développée, celle d'un écran réactif au contact du doigt. En effet, les notions d'immédiateté, d'absence de friction, d'interaction, de tactile, sont travaillées, si l'on peut dire, de l'intérieur par une pensée de ce que doit être le geste. La pensée du «tactile» implique une concordance, entre le geste et ce qui est restitué à l'écran. C'est-à-dire que l'idéal du «bon design» et du «bon écran » consisterait en une continuité entre le geste et sa réalisation ou sémiotisation à l'écran. L'horizon de cette idéologie est finalement la disparition, paradoxalement, du corps de l'utilisateur : comme tout media «classique », il y a une disparition de ce qui permet la médiation, ici, le doigt en particulier et le corps en général. Cette idéologie repose ainsi sur une sorte de pureté, d'idéal de la manipulation et de l'intention qui consacre le rôle des designers d'expérience et d'interface en architecte de l'enchantement de l'usager.

Les manières de faire du design proposent en plus une idéologie qui tend à objectiver le corps, en faire une ressource. Ces écrans d'abord tireraient parti d'une activité nécessaire des mains. Darian Leader (2017), psychanalyste, explique que s'occuper les mains serait essentiel à l'humain. La rencontre fréquente entre les mains et l'écran serait inévitable (tout en étant stimulée) car il leur proposerait de s'occuper. Il y aurait une disposition à l'écran. En cela, le corps est investi comme corps touchant. Mais le corps est également investi comme corps pensant, se voit attribuer un rôle dans sa capacité à avoir certaines habitudes corporelles. Les procédures de manipulation de l'écran en effet font, dans les exemples cités, appel à la mémoire corporelle, sensorielle et culturelle, de l'usager. Les gestes métaphorisés renvoient à des pratiques habituelles, cherchent à stimuler une mémoire des formes (Souchier, 2012b) et des procédures, ou à reproduire des gestes déjà ancrés, plutôt qu'un raisonnement sur l'activité de l'écran. Cette "pensée » du corps n'est possible que par la fréquentation des écrans qui a permis l'incorporation de ces procédures, selon le processus d' « intussusception » décrit par Marcel Jousse (1974) (du latin, intus, dedans, et suscipere recevoir). Leur fréquentation les a rendus familiers, "co-naturels » et l'on peut les faire sans y penser, ou en pensant ou en faisant autre chose, comme si le corps prenait en charge la majorité de l'activité - c'est le propre de la «mémoire de l'oubli» (Souchier, 2012b).

De tout cela, il ressort, en creux, une représentation du corps qui justifie que l'on parle de « viande » plutôt que de « chair ». La figure 1 fonctionne en effet comme une métaphore (du traitement de l'usager par l'application Tinder), mais aussi, à un second niveau, manifeste, presque malgré elle, la façon dont le design et les constructeurs logiciels traitent le corps. On peut en effet distinguer les propriétés associées à la chair, et celles associées à la viande. Pour Merleau-Ponty la « chair » est ce qui met en relation « ce que l'on nomme le psychique et le corporel» (Cotten, 2000). On sait depuis ses travaux que la pensée émerge d'une chair mise en mouvement, affectée. On pourrait supposer que c'est sur cette approche d'un corps mis en activité, en micros-mouvements, que s'appuie l'idéologie des écrans tactiles, donnés à manipuler et à interpréter. Pourtant, l'usage qui est fait du corps n'est pas tant un usage de chair que de viande telle que le conceptualise Gilles Deleuze à partir de la peinture de Francis Bacon. De son analyse, il ressort une approche de la viande comme souffrance, «l'idée de la sensation dans sa dimension négative, qui affecte en mal» (Girard, 2016). La viande s'oppose également à la chair en ce qu'elle est dénuée de toute forme d'idée. Alors que la chair marque un basculement dans le proprement humain, vers l'interprétation, la viande, à l'inverse, renvoie à une animalité - voire une bestialité. Sans affirmer que le corps de l'usager n'est que souffrance, on peut constater que la sensibilité, qui traverse le régime de l'illusion et qui participe à l'objectivation du corps, est mobilisée dans sa dimension négative.

Les écrans tactiles tendent ainsi à faire émerger l'idée d'un corps dénué de ses capacités interprétatives et de sensorialité « fiable». En trompant les sens et en participant à techniciser le corps de l'usager, à prétendre faire disparaitre son corps, ces écrans manifestent une idéologie qui, dans cette opposition entre chair et viande, tend à faire du corps un « simple » morceau de viande (humaine).

\footnotetext{
${ }^{16}$ Comme le souligne déjà Mateo Treleani, « Le paradigme de l'ordinateur, [...] consiste en une praxéologie : pratiques, actions. Si au cinéma le dispositif nous met dans la condition d'un spectateur qui a le contrôle du voir mais non pas du faire, avec l'ordinateur nous sommes des usagers : actifs et même interactifs, nous agissons et nous manipulons les contenus qui nous sont présentés » (Treleani, 2014)
} 


\section{Une sensibilité toujours plus forte au corps de l'utilisateur masquée par le discours de la « prothèse »}

Au regard du fonctionnement des dispositifs contemporains, ce n'est pas qu'à la peau, mais au corps entier, que l'écran est sensible. C'est pourquoi la centralité du contact (sur lequel insiste le terme " tactile ») doit être remise en question : c'est avec le corps en mouvement que s'écrit le numérique. La représentation tenace de l'écran comme prothèse ${ }^{17}$ rend opaque un fonctionnement qui repose sur l'écriture et la captation du corps, qui seraient sans doute jugées inacceptables par l'usager s'il en avait pleinement conscience. C'est pourquoi nous proposons de revenir plus en détail sur les imaginaires de l'écran, et en proposons d'en faire, pour terminer, la critique.

\subsection{Rejeter la centralité du contact}

Il semble pour finir que la couche de manipulation disparaisse dans certains cas, que l'écran parvienne à s'en passer. Ou plutôt, que ce n'est plus uniquement au geste à sa surface que les écrans contemporains répondent. Dans les dispositifs usuels (Facebook, Instagram, LinkedIn, GoogleMap, Tinder...) certaines informations à l'écran ne relèvent pas d'une manipulation réalisée par l'usager mais sont tout de même enregistrées : la géolocalisation, les distances parcourues, la vitesse moyenne d'une course, le nombre de marches montées, les pulsations cardiaques, les lieux visités, etc. Des informations que l'utilisateur n'a pas déclarées par activation de l'écran, en le touchant ou en l'effleurant, mais qui ont été captées, se sont " écrites » pendant l'activité. Cela est exemplaire des capacités de l'écran à être sensible à autre chose qu'au doigt.

C'est le corps tout entier qui écrit pour l'écran, ses mouvements et ses déplacements; l'écran s'écrirait avec les mouvements du corps. L'idée du tactile doit changer : la "peau » de l'écran s'étend désormais au-delà du contact du doigt. Cette observation demande de s'intéresser au devenir des interactions tactiles et à leurs transformations par le dispositif. S'il y a des informations qui s'écrivent à l'écran que l'utilisateur n'a pas enregistrées volontairement, alors, c'est bien que l'écran ne donne à voir et à manipuler que ce qui a été décidé en amont. À l'inverse, certaines données enregistrées par le dispositif ne sont pas toutes retransmises à l'usager. En fait, toute activité du corps peut potentiellement être enregistrée pour faire fonctionner le dispositif et engager la rétention de l'usager, mais aussi revendue sous la forme de données. Il ressort de cela que l'usager est mis à l'écriture, sans s'en rendre compte et cela semble indispensable pour le bon fonctionnement du dispositif. En perdant le contact avec le tactile, l'usager perd aussi la maîtrise de ce qu'il produit et de ce qui se passe « derrière l'écran ».

Tout cela semble reposer notamment sur la dimension affective des écrans, entretenue par les nombreux discours qui les accompagnent. Il y a quelque chose de plaisant et de séduisant à les voir s'animer, et tout est fait pour « enchanter » (Winkin, 1996) via la manipulation. Mais aussi, ils apportent des gratifications inédites à l'usager : des informations sur des performances, des indicateurs de popularité, des bonnes pratiques de marches, etc. (selon les dispositifs). Sans entrer dans le détail des informations enregistrées sans contact direct, elles visent à apporter des informations censées intéresser l'usager, lui apporter un plus au quotidien. En ce sens, l'écran reflète à nouveau la projection et les conceptions subjectives de ses concepteurs, des «anticipations» de ce qu'ils projettent être attendu et utile. Cela apporte des éléments pour la compréhension des rapports à l'écran et explique que les usagers acceptent assez « facilement » de jouer le jeu des dispositifs, sans relever le fait que les écrans sont capables d'écrire sans leur signature propre. Pour reprendre les termes de Gustavo Gomez-Mejia, qui s'intéresse moins aux écrans qu'aux dispositifs numériques, c'est une double « rétribution », qu'obtient l'usager en échange de sa manipulation : un plaisir des sens (le « tactile » en lui-même) et un plaisir intellectuel (les informations redistribuées). C'est sur cette double rétribution que se joue la «contribution» de l'usager pour son usage de ces écrans, comme cela est décrit par Gomez-Mejia sur Facebook :

«[D]es jeux métaphoriques sont cruciaux pour rendre lisible conceptuellement le fonctionnement des sites aux yeux des internautes, pour favoriser leur implication identitaire dans une structure imaginée de manière plus anthropomorphe que scripturaire, ainsi que pour encourager des rituels de productivité à l'écran ». (Gomez-Mejia, $2016: 51$ ).

Avec ce couple « rétribution » / « contribution », il explique que ces dispositifs industriels proposent des contenus qui reposent sur des métaphores (comme le fait de se constituer un profil) qui permettent à l'usager de comprendre ce qu'il est possible de faire; de l'engager et de lui faire adopter le service. La métaphore masque l'activité d'écriture qui lui est demandée, la rend invisible, et favorise au quotidien la production de traces et de représentations d'informations monétisables à son égard. C'est ce qui se passe au niveau-même de la manipulation de l'écran. L'utilisateur accepte en premier lieu cette manipulation car elle repose essentiellement sur des métaphores qui ne rendent pas visible le travail d'interprétation et d'écriture. Mais aussi, il n'interroge pas son extension au-delà du doigt car il en tirerait un plaisir et des gratifications nouvelles. Les applications dites de monitoring de soi en sont un exemple : avec la mise en écriture d'informations enregistrées de façon automatique, par un mouvement du corps, quelque chose de plus est offert à l'utilisateur. Il obtient des informations jusque-là difficilement accessibles sur ses performances, des mesures de son quotidien. Ces informations sont quelque fois même «cliquables » pour leur détail ; en ce sens, «manipuler», des

${ }^{17}$ Autour de l'écran comme prothèse, voir M. Carbone, Philosophie-écrans, Vrin, 2016. 
données sur soi, a quelque chose de satisfaisant et cela participe à rendre littéralement indolore la monétisation ultérieure de ces données.

Pour être acceptés, ces écrans jouent sur une capacité de l'usager à être affecté et rentabilisent cette affectivité. Le terme de monitoring de soi qui cherche à nommer ces pratiques d'aperçu de performance promet en lui-même de se « manipuler », faisant de l'écran un adjuvant utile et de bien-être. Ce type d'écrans et son fonctionnement est iconique de l'"économie affective» de ces services décrite par Camille Alloing et Julien Pierre : «les différentes formes d'exploitation des affects numériques / numérisés, de leur circulation et de leur mesure » (Alloing et Pierre, 2017: 32). En stimulant affectivement le corps et l'activité cognitive de l'usager, ces écrans participent pleinement de cette économie.

\subsection{Des imaginaires qui font diversion}

Revenons, pour terminer, sur les imaginaires de l'écran et observons quel écran ils construisent. Il s'avère que ces discours préparent, en amont de l'usage, à l'illusion d'une continuité entre le corps et l'écran, à avoir affaire à une machine un peu magique. Diversions fictionnelles et sémiotiques, ces représentations contribuent à produire une forme de confiance et de fascination sociale. Elles nient et rendent complètement opaque la sensibilité exacerbée par les écrans au corps humain, à ses mouvements, et aux enjeux économiques et de pouvoir qui les traversent.

Cette fascination pour le tactile est encadrée, dans une certaine mesure, par les discours issus notamment du champ de la science-fiction. Les romans, les productions cinématographiques et aujourd'hui les séries en proposent une vision magnifiée et souvent exagérée dans ses capacités. Ce type de discours accompagne les innovations de l'écran, prépare sa réception, engage certaines postures et attitudes face à l'objet. Enjeu discursif, l'écran est fantasmé, ancré dans des imaginaires et objets de représentations qui en conditionnent ses appropriations. Le film Minority Report est un bon exemple des représentations de l'écran par le discours de la science-fiction. Sorti en 2002, adapté de l'œuvre du romancier Philip K. Dick, ce film présente une vision enchantée des écrans mis en scène comme des objets puissants. Ils se manipulent du bout du doigt, d'un effleurement de la surface, sont sublimés, ils sont transparents, extrêmement rapides et performants. Ce qui est mis en scène à travers ces représentations, c'est une forme de plaisir de l'agentivité de l'usager, du fait de voir l'écran réagir sous le doigt, un plaisir lié à la réponse au geste et à l'œil de la surface transparente. Le spectateur au moment de la sortie du film, et quelques années après, pouvait éprouver une forme de satisfaction à voir l'écran répondre à la moindre commande (à la sortie du film, l'écran du grand public n'est pas encore tactile), mais en plus, les formes représentées sont idéalisées par une esthétique futuriste. Ce type de mise en scène de l'écran ancre un plaisir attendu dans la manipulation : un écran serait bien quand il répond immédiatement au moindre de ses gestes. À côté de cet imaginaire fantasmatique, esthétique et plaisant de l'écran, une autre représentation s'impose : il est fréquemment représenté dans un prolongement contigu et continu avec l'humain. Cela passe par la figure de la prothèse, mais aussi par la mise en scène d'une continuité fine entre le corps et la technique, jusqu'à se passer d'écran. On trouve ce motif à nouveau dans l'œuvre de K. Dick et dans l'adaptation par la série Philip K Dick's Electric dream de ses œuvres. Dans l'épisode 6 de la première saison, on voit un dispositif attaché au poignet d'un personnage et lui permettant d'interagir avec une représentation de type écranique projetée face à lui - sans surface -, directement avec ses doigts. L'écran se rêve également par son absence, tout en étant présent. Ce type de discours, d'une interface sans médiation, est typique du constat fait par Etienne Candel, qui observe que les discours d'accompagnement «constitue[nt] en prolongement des corps et en outils capables d'augmenter les capacités des usages » (Candel, 2019) les dispositifs mobiles.
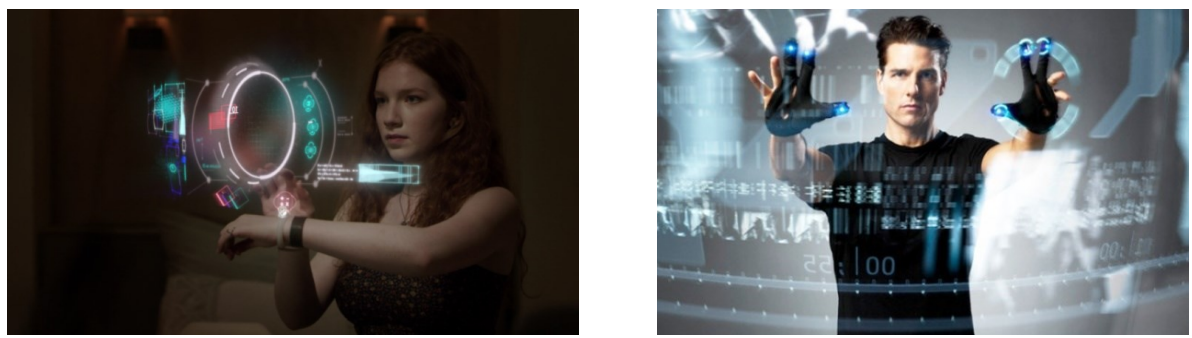

Figure 4. À gauche, la manipulation magique de Safe around-Philip K Dick's electric dreams $1^{E} 6$; à droite, la manipulation " plaisante » de Minortity Report. Sources : https://io9.gizmodo.com/electric-dreams-best-episode-puts-all-my-parental-nigh1822647491 - consulté le 11/11/2020 ; http://cinemachoc.canalblog.com/archives/2016/09/03/33749171.html - consulté le $11 / 11 / 2020$.

Ces discours de la prothèse et du plaisir de la manipulation de l'écran - et de ce qui ressemble à ce type d'expérience accompagnent la commercialisation, la circulation et l'appropriation des écrans tactiles et servent de référents pour les penser. L'expérience de ces dispositifs n'est pas vécue ni perçue de façon neutre, mais, au contraire, tout objet ou toute 
action s'accompagne de représentations qui la travaillent, qui en préparent l'usage et qui en colorent les pratiques ${ }^{18}$. Sa réception serait prédéterminée par ces représentations vives et présentes, que le paysage médiatique et culturel rend sensible et entretient : elles instituent d'emblée une approche fascinée, enjouée, agréable de l'écran. Les imaginaires jouent comme un cadre de compréhension et de jugement, et cela est plus que jamais vrai dans le cadre d'artefacts aux fonctionnements techniques opaques.

Ces discours sur la prothèse font diversion. Quelque fois critiques, ils échouent tout de même à cerner l'actualité de la question en se focalisant sur les enjeux d'hybridation. À l'opposé de cette idée répandue que l'être humain est voué à ne faire qu'un avec la technique, la prothèse se trouverait dans l'externalisation et la constitution en mémoire du numérique (Souchier et al. 2019 : 89), "sans contact» mais "proche du corps ». Il faut enfin revenir sur les termes évoqués, de « tactile », de « fluidité », d' « intuitivité ». Le rapport entre l'objet matériel qu'est l'écran et la captation de données est nié par le nom même de l'objet : tactile passe sous silence cette activité de captation. Alors que les écrans ont changé de formes, de fonctions, d'usages, le même terme est resté. L'écran des smartphones n'a plus grand-chose à voir avec le synthétiseur électronique mis au point en 1953 par un musicien canadien pour contrôler le timbre et le volume de son instrument, que l'encyclopédie en ligne Wikipédia désigne comme le premier écran tactile. Le terme entretient donc une confusion sur le fonctionnement de l'écran, et en masque complètement les enjeux. Le vocabulaire métaphorique questionné précédemment constitue une diversion efficace de ce qu'il se passe avec les écrans: en focalisant l'attention sur l'interface, en flattant l'utilisateur et en assumant l'objectif de lui rendre la manipulation agréable et dans un prolongement de son corps, il détourne l'attention des changements et des évolutions de l'objet.

À force d'innovations, de la possibilité de connecter l'écran à des périphériques nouveaux et variés, toujours plus précis dans les formes d'enregistrement possibles, l'écran contemporain n'a plus rien des premières interfaces tactiles. Dans le foisonnement des discours autour de l'écran, l'enjeu de ses transformations et de ses appareillages disparait : l'attention est tournée vers le développement technique de l'interface et c'est dessus que portent les imaginaires de l'écran.

\section{Conclusion}

Les écrans tactiles participent d'une transformation idéologique de la chair de l'être humain en viande. Ils enregistrent sa position sur sa surface, et sont désormais capables de la traiter à distance. Contre la pensée magique de l'écran, nous avons déterminé les conditions de réussite d'une activité tactile : la rencontre entre un écran et une main articulée animée par un esprit humain. La prothèse ensuite est affaire d'illusion entretenue grâce à des procédés rhétoriques culturels, visuels et symboliques. Enfin, il y a transfert de propriétés, entre la main, le corps et l'écran. L'écran est entièrement pensé autour de la main. On retrouve dans l'écran toutes les qualités de cet organe polyvalent. L'écran, c'est le corps. Mais aussi, le corps de l'usager est le lieu de l'interprétation et de l'incorporation de l'écran. C'est à partir de sa perception qu'il donne sens à l'écran. Enfin, le corps écrit désormais l'écran. Le corps, c'est aussi l'écran (Dalmasso, 2018).

Mais gardons-nous de réduire l'épaisseur du phénomène sémio-technique dans cette double relation d'équivalence. L'étude a montré la force d'institutionnalisation des guidelines, guides de bonne pratiques et discours spécialisés pour la construction d'interface. Ceux-ci participent d'une institution entre le signal et le signe à l'écran. Les constructeurs et concepteurs d'interfaces, ergonomes, designers d'expériences, s'appliquent à construire une illusion de continuité entre la pensée, le geste et l'écran, et à la renforcer par un travail, sur ce qu'il renvoie, mais aussi sur ce qu'il propose. Ils s'appuient sur des savoir-faire et des jeux techno-sémiotiques venant activer les sens. Poser le phénomène sur le plan technique a permis d'éviter le vocabulaire métaphorique de rendu de l'interface. Parler de « tactile », de « réactivité » et de « fluidité » empêche de voir à quel point ce phénomène est construit à un niveau technique par des acteurs ayant certaines conceptions. Lorsque l'on actualise un écran, on retrouve les conceptions des concepteurs, largement structurantes. « Si auparavant le spectateur d'un film convoitait et essayait d'imiter le corps de la vedette ; l'utilisateur d'un ordinateur est désormais invité à suivre la trajectoire mentale du concepteur des nouveaux médias » (Manovich, 2001 : 149), et, pourrait-on rajouter, avec les dispositifs tactiles, une manière d'interpréter, de penser, de percevoir, et de se tenir dans le monde.

Nous avons enfin cherché à souligner la nécessité d'éviter de parler de «tactile ». L'enjeu est épistémologique et politique. Pour produire un savoir sur ces technologies, on doit remettre en question ce terme, et ceux qui l'accompagnent, car ils instaurent d'emblée une relation inégale face à la technique. Articuler plusieurs approches de l'écran s'est avéré heuristique. L'approche technique a permis de comprendre son fonctionnement, celle par l'anthropologie du geste de comprendre comment il était fait pour la main et en vue de la main. Enfin, l'approche par l'étude des écrits d'écran a permis d'intégrer une approche contextualisée de ces écrans avec leurs enjeux de captation et de valorisation. Penser le " tactile» en termes d' "enregistrement» et d' "écriture » a ouvert la notion et a montré l'intérêt de la conception à entretenir l'impression de magie et de prothèse.

\footnotetext{
${ }^{18}$ Ce type de processus a été mis en évidence par Yves Jeanneret, Philippe Breton ou Patrice Flichy en sciences de l'information et de la communication.
} 


\section{Références}

Alloing Camille, Julien Pierre. (2017). Le web affectif : une économie numérique des émotions. Paris : INA éditions.

Candel Etienne. (2020). Les nouveaux outils du pouvoir : tours et atours technologiques de l'autorité, Quaderni, 99100 , p. $137-150$.

Cotte Dominique. (2004). Écrits de réseaux, écrits en strates. Sens, technique, logique, Hermès, La Revue, 2 (39), p. 109-115.

Cotten Jean-Pierre. (2000). L'“expérience’ de la chair chez le dernier Merleau-Ponty, Philosophique, 3, p. 19-35.

Davallon Jean, Després-Lonnet Marie, Jeanneret Yves, Le Marec Joëlle \& Souchier Emmanuël. (2003). Lire, écrire, récrire: Objets, signes et pratiques des médias informatisés. Paris: Éditions de la Bibliothèque publique d'information.

Hustache-Godinet Hélène. (1993). Quelques néologismes du lexique informatique. Bulletin de 1'EPI (Enseignement Public et Informatique), Association EPI, p. 53-69. [https://edutice.archives-ouvertes.fr/edutice$\underline{00001116 / \text { document }]}$

Girard Maxime. (2016). Forces et sensations dans la peinture de Francis Bacon référence : Francis Bacon, logique de la sensation; skira », Philosophique, 19, [http://journals.openedition.org/philosophique/948].

Le Breton David. (2006). La saveur du monde. Une anthropologie des sens. Paris : Métailié, coll. « Traversées ».

Jeanneret Yves. (2014). Critique de la trivialité. Les médiations de la communication, enjeu de pouvoir, Paris : Editions Non Standard.

Jousse Marcel. (1969). L'anthropologie du geste I. Paris, France : Les Éditions Resma.

Leader Darian. (2017). Mains, Ce que nous faisons d'elles et pourquoi, Paris : Albin Michel.

Leroi-Gourhan André. (1965). Le geste et la parole, vol. 1. La mémoire et les rythmes. Paris : Albin Michel.

Mcluhan Marshall. (1977). Pour comprendre les médias, trad. fr. 1968, rééd. Paris : Seuil, coll. « Points essais ».

Merleau-Ponty Maurice. (1945). Phénoménologie de la perception, Paris : Gallimard.

Merleau-Ponty Maurice. (1964). L'eil et l'esprit, Paris : Gallimard.

Miège Bernard. (2015). Contribution aux avancées de la connaissance en Information-Communication, Bry-surMarne : INA Éditions.

Ouvrier-Bonnaz Régis. (2014). La libération de la main d'André Leroi-Gorhan, Perspectives interdisciplinaires sur le travail et la santé, 16-3. [http://journals.openedition.org/pistes/3629]

Platon. Phèdre, Paris : Garnier-Flammarion.

Souchier Emmanuël, Candel Étienne, Gomez-Mejia Gustavo, Jeanne-Perrier Valérie. (2019). Le numérique comme écriture. Théories et méthodes d'analyse. Paris : Armand Collin.

Souchier Emmanuël. (2012a). La « lettrure» à l'écran. Lire \& écrire au regard des médias informatisés, Communication \& langages, 2012/4, 174, p. 85-108.

Souchier Emmanuël. (2012b). La mémoire de l'oubli : éloge de l'aliénation. Pour une poétique de «l'infraordinaire " ", Communication \& langages, 2012/2, p. 3-19 ;

Souchier Emmanuël. (2004). Mémoires - outils - langages. Vers une « société du texte »?. Communication \& Langages, 139, p. 41-52.

Souchier Emmanuël. (1996). L'écrit d'écran, pratiques d'écriture \& informatique. Communication et langages, $\mathrm{n}^{\circ} 107$, 1 er trimestre. p. 105-119.

Treleani Matteo (2014). Dispositifs numériques, Actes Sémiotiques, 117, 2014. [https://www.unilim.fr/actessemiotiques/5035]

Winkin Yves. (1996). Anthropologie de la communication De la théorie au terrain. Bruxelles : Éditions De Boeck Université.

\section{Documentation}

http://interfacetactile.com/ecran-tactile-capacitif - consulté le 08/05/2020.

https://www.futura-sciences.com/tech/definitions/technologie-ecran-tactile-539/ - consulté le 08/05/2020.

https://www.usabilis.com/qu-est-ce-que-le-skeuomorphisme/ - consulté le 08/05/2020. 